\title{
Enzymatic modification of grapeseed (Vitis vinifera L.) oil aiming to obtain dietary triacylglycerols in a batch reactor
}

\author{
Natalia Bassan $^{\mathrm{a}}$, Rafael Hatanaka Rodrigues ${ }^{\mathrm{b}}$, Rubens Monti ${ }^{\mathrm{a}}$, Carla Tecelão ${ }^{\mathrm{c}, \mathrm{d}}$, \\ Suzana Ferreira-Dias ${ }^{\mathrm{d}}$, Ariela V. Paula ${ }^{\mathrm{a}, *}$ \\ a State Universidade Estadual Paulista (Unesp), Faculdade de Ciências Farmacêuticas, Araraquara/ São Paulo State University (Unesp), School of Pharmaceutical \\ Sciences, Araraquara, Brazil \\ ${ }^{\mathrm{b}}$ State Universidade Estadual Paulista (Unesp), Institute of Chemistry, Organic Chemistry Department - Center for Monitoring and Research of the Quality of Fuels, \\ Biofuels, Crude Oil and Derivatives e CEMPEQC, Araraquara, SP, Brazil \\ ${ }^{\mathrm{c}}$ MARE - Marine and Environmental Sciences Centre, ESTM, Instituto Politécnico de Leiria, 2520-641 Peniche, Portugal \\ d Universidade de Lisboa, Instituto Superior de Agronomia, LEAF, Linking Landscape, Environment, Agriculture and Food, Tapada da Ajuda, 1349-017 Lisbon, Portugal
}

\section{A R T I C L E I N F O}

\section{Keywords:}

Acidolysis

Batch reactor

Grape seed oil

Lipase

Structured lipids

\begin{abstract}
A B S T R A C T
Structured lipids (SL) are chemically or enzymatically modified oils and fats with respect to their original fatty acid composition or position in acylglycerols. These compounds present improved functional or nutraceutical properties. The present work aimed at the enzymatic synthesis of SL, MLM-type dietary triacylglycerols, that is, those with medium chain fatty acids (M) at the $s n-1$ and $s n-3$ positions, and long chain fatty acids (L), in the internal position of the triacylglycerol. Grapeseed oil was selected based on its composition rich in unsaturated fatty acids, principally linoleic acid. This oil was submitted to batch acidolysis with medium chain fatty acids (caprylic or capric) in solvent-free media. Reactions were catalyzed by different immobilized commercial lipases, namely: Lipozyme TL IM ${ }^{\circledast}$ (Thermomyces lanuginosa lipase), Lipozyme RM IM ${ }^{\circledast}$ (Rhizomucor miehei lipase) and Novozym $435^{\circ}$ (Candida antarctica lipase B). The incorporation degree (ID) ranged from $23.62 \pm 1.34$ to $34.53 \pm 0.05 \mathrm{~mol} \%$, after $24 \mathrm{~h}$ reaction at $45^{\circ} \mathrm{C}$, using a molar ratio (MR) fatty acid:oil of $2: 1$. The best results were obtained using capric acid and Lipozyme $\mathrm{RM} \mathrm{IM}^{\circ}$ lipase $(34.53 \pm 0.05 \mathrm{~mol} \%)$. In the experimental design, the influence of MR and temperature on ID were evaluated. ID increased with MR and T and was fitted to a saddle-like surface.
\end{abstract}

\section{Introduction}

Modified oils and fats with improved functional and/or nutritional properties have received special attention, due to the importance of the diet on the promotion and maintenance of health. These novel fats and oils, known as Structured lipids (SL), are usually chemically or enzymatically synthesized by modification of triacylglycerols with respect to their original fatty acid composition or fatty acid position in glycerol backbone (Cao et al., 2013; Liu et al., 2015).

Within Structured lipids, the class of dietary triacylglycerols (TAGs) is highlighted, which are synthesized for specific nutritional applications (Cao et al., 2013). This class of lipids has lower caloric value (5-7 kcal/g) than conventional oils and fats $(9 \mathrm{kcal} / \mathrm{g})$. This is because they have a lower amount of long chain fatty acids at the $s n-1$ and $s n-3$ positions, which consequently increases their solubility. During digestion, the medium chain fatty acids, released from TAG, are metabolized as rapidly as glucose and, therefore, they are not stored as fat, with weight control benefit (Ferreira-Dias, Sandoval, Plou, \& Valero, 2013; Morales-Medina, Munio, Guadix, \& Guadix, 2017). The interest in these TAGs has recently increased due to its main nutritional characteristic. They can be administered orally, through probes or mixed with other foods, in the treatment of patients who present metabolic difficulties related to diseases such as AIDS, cystic fibrosis and cirrhosis. They also are used in severely malnourished patients (Liu et al., 2015).

The enzymatic synthesis of Structured lipids is normally catalyzed by regio-selective $s n-1,3$ lipases, which allows synthesizing compounds that are not possible to obtain chemically because chemical catalysts are non-regioselective, acting at random in TAGs (Li et al., 2017; Rehman, Wang, Bhatti, Bilal, \& Asgher, 2017).

The aim of this work was to synthesize dietary triacylglycerols of the MLM-type (medium: long: medium), triacylglycerols containing medium chain fatty acids (M) at the $s n-1$ and $s n-3$ positions and long

\footnotetext{
* Corresponding author.

E-mail address: ariela@fcfar.unesp.br (A.V. Paula).
} 
chain fatty acids (L) in position $s n$-2. In this context, grapeseed oil was selected as source of polyunsaturated long-chain fatty acids at position $s n-2$ in TAGs, due to its high nutritional value. It is also derived from waste generated from the production of juice and wine (Da Porto \& Natolino, 2017). Grapeseed oil has a high content of unsaturated fatty acids, such as essential linoleic acid (58-78\%), which is higher than in other oils such as corn (34-65.6\%) and soybean (48-59\%) oils (FAO, 1999). In addition, it has a high amount of antioxidant compounds, such as tocopherols (vitamin E) and tocotrienols (Raeisi, Tajik, Aliakbarlu, Mirhosseini, \& Hosseini, 2015). The use of grapeseed oil for producing MLM-type TAGs by acidolysis with caprylic or capric acids, in solvent-free media, catalyzed by non-commercial $s n-1,3$ regioselective recombinant Rhizopus oryzae lipase and Carica papaya lipase self-immobilized in papaya latex was recently reported (Costa et al., 2018).

The objective of this work was to optimize the enzymatic synthesis of dietary triacylglycerols of the MLM-type by batch acidolysis of grapeseed oil with medium chain fatty acids, caprylic (C8: 0) or capric (C10: 0) in solvent-free media, employing commercial immobilized lipases as catalysts.

\section{Material and methods}

\subsection{Material}

\subsubsection{Biocatalysts}

The commercially immobilized enzymes used as biocatalysts were: Lipozyme TL IM ${ }^{\circledR}$ (Thermomyces lanuginosa lipase), Lipozyme RM IM ${ }^{\circledR}$ (Rhizomucor miehei lipase) and Novozym $435^{\circ}$ (Candida antarctica lipase B), kindly provided by Novozymes A/S (Bagsvaerd, DENMARK). Porcine pancreatic lipase was purchased from Sigma-Aldrich.

\subsubsection{Raw material}

Grapeseed (Vitis vinifera L.) oil was obtained by cold pressing and filtration from Distriol (São Paulo, BRAZIL), Capric acid was purchased from Merck (Darmstadt, GERMANY), and caprylic acid from Sigma Aldrich (São Paulo, BRAZIL).

\subsection{Methods}

\subsubsection{Characterization of grapeseed oil}

The acid value (Ca 5a-40 method) and peroxide value (Method Cd 8b-90) were determined according to the official methods of the American Oil Chemists' Society (AOCS, 2011). Fatty acid composition was determined by gas chromatography, according to the American Oil Chemists' Society Method Ce 2-66 (AOCS, 2011).

\subsubsection{Determination hydrolytic and esterification activities of the biocatalysts}

The hydrolytic activity of the biocatalysts was assayed by the titration method using an olive oil emulsion (Paula, 2012; modified). The esterification activity was quantified by the consumption of oleic acid according to the method described by Pinto, Freire, and Pinto (2014). One unit of activity (U) was defined as the amount of enzyme that released $1 \mu \mathrm{mol}$ of fatty acid per minute under the assay conditions.

\subsubsection{Acidolysis reaction for the synthesis of dietary triacylglycerols (MLM) from grapeseed oil}

Acidolysis reactions between the vegetable oil (grapeseed) and the medium-chain fatty acids (caprylic or capric) were carried out in a jacketed cylindrical glass reactor (internal diameter of $3 \mathrm{~cm}$, height of $6 \mathrm{~cm}$ ), operating in a batch regime. The reactions occurred at a temperature of $45^{\circ} \mathrm{C}$, with constant agitation of $400 \mathrm{rpm}$, molar ratio (MR) fatty acid:oil of 2:1 and enzyme load of $5 \%$ (relative to the total mass of medium). The MR used in these experiments corresponds to the stoichiometric value for acidolysis catalyzed by $s n-1,3$ regioselective lipases. Samples were collected at 0 time (oil without modification) and after $24 \mathrm{~h}$ reaction, and analyzed with regard to acidity, peroxide value, fatty acid composition and fatty acid composition at the $s n-2$ position.

\subsubsection{Modeling acidolysis reaction}

After the selection of the best reaction system (oil: acid and lipase), the influence of the molar ratio (MR) oil:capric acid and temperature (T) was evaluated in the acidolysis reaction, aiming to model acidolysis and optimize the reaction conditions that maximize the incorporation of capric acid. A Central Composite Rotatable Design (CCRD), consisting of a factorial matrix $2^{2}$, (levels $(+1)$ and $(-1)$ ), complemented with four tests of the axial points and the repetition of the central point, was used. The experimental conditions tested (temperature: $41-69{ }^{\circ} \mathrm{C}$; MR: 1:1 to 7:1) were selected from similar works on acidolysis reactions aimed at MLM production in solvent-free media, catalyzed by the same immobilized enzymes (Caballero, Soto, Olivares, \& Altamirano, 2014; Nunes, Pires-Cabral, \& Ferreira-Dias, 2011).

The incorporation degree of capric acid into grapeseed oil was assumed as the response. The experimental results were analyzed using the Statistica program, Statsoft, Tulsa, version 6. The polynomial model adjusted to the experimental results was validated by performing independent tests with different values of temperature and molar ratio within the experimental range considered in the study.

\subsubsection{Separation and methylation of triacylglycerols}

After $24 \mathrm{~h}$ reaction, $1.5 \mathrm{~g}$ of the sample were weighed. Then, $30 \mathrm{~mL}$ of hexane and $10 \mathrm{~mL}$ of the hydroalcoholic solution (30\% ethanol) of potassium hydroxide $(0.8 \mathrm{M})$ were added. The mixture was kept under stirring and then allowed to stand for phase separation. The organic (upper) phase was collected, repeating the triacylglycerol extraction with hexane $(10 \mathrm{~mL})$ again. The solvent was extracted in a rotavaporator $\left(\mathrm{P}=130 \mathrm{mbar}\right.$ and $\left.\mathrm{T}=40^{\circ} \mathrm{C}\right)$ and the TAGs were stored at $8{ }^{\circ} \mathrm{C}$ until further analysis (Wang, Xia, Xu, \& Xie, 2012). Methylation was performed according to ISO 12966-2 (2011), after modification. Approximately, $50 \mathrm{mg}$ of the purified triacylglycerols were weighed and $2 \mathrm{~mL}$ of a sodium hydroxide methanolic solution $(0.2 \mathrm{M})$ was added and the mixture was heated at $80^{\circ} \mathrm{C} / 15 \mathrm{~min}$ under vigorous stirring. Then, $2 \mathrm{~mL}$ of a methanolic solution of sulfuric acid $(1 \mathrm{M})$ was added, repeating the steps of heating and stirring (once time). One $\mathrm{mL}$ of a saturated $\mathrm{NaCl}$ solution and $2 \mathrm{~mL}$ of hexane were added. The solution was vortexed and then kept at rest for phase separation. The upper (organic) phase was removed and the extraction procedure with hexane $(2 \mathrm{~mL})$ was repeated. The hexane was removed at $40^{\circ} \mathrm{C}$ in the rotavapor and the fatty acids methyl esters were stored at $6^{\circ} \mathrm{C}$ for further analysis.

\subsubsection{Fatty acid composition of structured lipids and incorporation degree (ID, \%)}

The fatty acid composition of the structured lipids obtained by acidolysis of grapeseed oil was determined by gas chromatography, according to the American Oil Chemists' Society Method Ce 2-66 method (AOCS, 2011), as reported for fatty acid composition of grapeseed oil (cf. 2.2.1.).

The incorporation degree (ID) was calculated according to equation (1) (Casas-Godoy, Marty, Sandoval, \& Ferreira-Dias, 2013).

$I D(\%)=\frac{M F A}{M T} * 100$.

Where: MFA is the number of moles of medium chain fatty acids (C8:0 or C10:0) in TAGs and MT is the number of total moles of fatty acids in TAGs.

\subsubsection{Fatty acid at the sn-2 position in triacylglycerols}

The fatty acid composition at position $s n-2$ of the TAGs was analyzed following the porcine pancreatic lipase (PPL) hydrolysis 
procedure according to Caballero et al. (2014), modified. One $\mathrm{mL}$ of Tris-HCL buffer (pH 8.0), $0.250 \mathrm{~mL}$ of a solution of bile salts $(0.05 \%$, w/ v), $0.1 \mathrm{~mL}$ of a calcium chloride solution (22\%), the TAGs (100 mg) and the porcine pancreatic lipase $(100 \mathrm{mg})$ were mixed. The reaction occurred at $40{ }^{\circ} \mathrm{C}$ for $30 \mathrm{~min}$. The hydrolysis was stopped with the addition of $1 \mathrm{~mL}$ hydrochloric acid (6M) and $2 \mathrm{~mL}$ of diethyl ether. After, the blend was centrifuged $(5000 \mathrm{rpm} / 10 \mathrm{~min})$ and the upper layer was collected and applied to a silica-gel thin layer chromatography (TLC) plate. The TLC plate was put into a chromatographic chamber containing the mobile phase, consisting of hexane, ethyl ether and acetic acid $(70 / 30 / 1 ; \mathrm{v} / \mathrm{v} / \mathrm{v})$. After elution, the plate was visualized by spraying with an alcoholic solution of the dye 2,7 dichlorofluorescein $(0.2 \%)$ under u.v. light at $366 \mathrm{~nm}$. The $s n-2$ monoacylglycerol band was scrapped off, methylated and analyzed by gas chromatography (c.f. 2.2.1.).

\section{Results and discussion}

\subsection{Characterization of grape seed oil}

\subsubsection{Physico-chemical characterization of oil}

The grapeseed oil used in this study has an acid value of $0.225 \pm 0.021 \mathrm{mg} \mathrm{KOH} / \mathrm{g}$ of oil and a peroxide value of $0.905 \pm 0.148 \mathrm{mEq} \mathrm{O} / 2 \mathrm{~kg}$ of oil. These results showed that the raw material is within the specified standards (FAO, 1999): $0.6 \mathrm{mg} \mathrm{KOH} / \mathrm{g}$ of oil for the acid value and $10 \mathrm{meq} \mathrm{O}_{2} / \mathrm{kg}$ of oil for the peroxide value.

\subsubsection{Fatty acid profile}

Table 1 shows the results of the fatty acid composition of the grapeseed oil. The oil has high amounts of unsaturated fatty acids (c.a. $78 \%)$. The essential linoleic acid (C18:2) is the major fatty acid (46.2\%) in our sample, followed by oleic acid (C18:1; 26.6\%). Palmitic (C16:0, $11.1 \%)$ and stearic acids (C18:0; 6.72\%) are also important saturated fatty acids in grape seed oil. The highly polyunsaturated fatty acids C18:3 and of C20:3, which are rather prone to oxidation, are present in relative low amounts (3.46 and $2.11 \%$, respectively).

Due to the presence of high amounts of these fatty acids, the use of grapeseed oil has been gaining prominence and has been applied in different industrial sectors, such as in the food and cosmetic industries (Glampedaki \& Dutschk, 2014; Durante et al., 2017).

The intake of oils rich in these fatty acids brings great benefits to human health, ie the consumption of oils with large amounts of oleic acid is associated with the production of oleoylethanolamide, a compound that promotes catabolism and helps satiety in food intake (Bowen et al., 2017). In addition, the consumption of this fatty acid helps in the prevention of cardiovascular diseases, decrease of LDL (low density proteins), suppression of tumorogenesis and improvement of inflammatory diseases (Dhakal, Jung, Chae, Shannon, \& Lee, 2014).

On the other hand, the consumption of essential linoleic acid is related to the reduction of carcinogenesis, atherosclerosis, diabetes, as well as promotion and aid in bone formation and also acts as an anti-

Table 1

Fatty acid composition of grapeseed oil.

\begin{tabular}{ll}
\hline Fatty acid & $(\%)$ \\
\hline C16:0 & 11.10 \\
C18:0 & 6.72 \\
C18:1 & 26.57 \\
C18:2 $18: 3$ & 46.25 \\
C20:3 & 3.46 \\
C22:0 & 2.11 \\
$\mathrm{U}^{\mathrm{a}}$ & 1.16 \\
\hline
\end{tabular}

${ }^{\mathrm{a}} \mathrm{U}=$ unidentified fatty acid.
Table 2

Hydrolytic and esterification activities and moisture content of Lipozyme RM $\mathrm{IM}^{\circ}$, Lipozyme TL IM ${ }^{\circ}$ and Novozym $435^{\circ}$.

\begin{tabular}{llll}
\hline Parameter & Lipozyme RM IM & $\begin{array}{l}\text { Lipozyme TL } \\
\text { IM }\end{array}$ & $\begin{array}{l}\text { Novozym } \\
435\end{array}$ \\
\hline $\begin{array}{l}\text { Hydrolytic Activity }(\mu \mathrm{mol} / \mathrm{gmin})^{\mathrm{a}} \\
\text { (U/g) }^{\mathrm{a}}\end{array}$ & $568 \pm 42.02$ & $\begin{array}{l}5678 \pm 262 .- \\
99\end{array}$ & - \\
$\begin{array}{l}\text { Esterification Activity }(\mu \mathrm{mol} / \mathrm{gmin})^{\mathrm{a}} \\
\text { Moisture (\%, d.w) }\end{array}$ & 5543 & - & 6286 \\
& 5.11 & 7.86 & 6.86 \\
\hline
\end{tabular}

${ }^{\text {a }}$ Mean \pm standard deviation.

inflammatory agent (Viladomiu, Hontecillas, \& Bassaganya-Riera, 2016; Yang et al., 2015).

Some studies report that the amounts of oleic acid in grapeseed oil can range from 10 to $22 \%$ and linoleic acid from 60 to $75 \%$ (Irandoost, Ebrahimi-Mameghani, \& Pirouzpanah, 2013; Mironeasa, Leahu, Codinã, Stroe, \& Mironeasa, 2010; Rombaut et al., 2014). According to the results presented in Table 1, the grapeseed oil used in the present study presents oleic and linoleic acid contents within the variation range indicated in the Codex Alimentarius (Codex, 1999). Differences in fatty acid contents reported in literature, are justified by several factors, such as grape variety, edapho-climatic conditions and agronomic practices used (Duba \& Fiori, 2015; Fiori et al., 2014).

\subsection{Hydrolytic and esterification activities of the biocatalysts}

The hydrolytic and esterification activity data of the biocatalysts, as well as their moisture content are presented in Table 2. Novozym $435^{\circ}$ only presents esterification activity in contrast with Lipozyme TL IM ${ }^{\circ}$, which only shows hydrolytic activity. However, Lipozyme RM IM presents both lipolytic and esterification activities. However, the esterification activity of this enzyme is almost 10 -fold its hydrolytic activity. The moisture content of these biocatalysts are not very different and therefore do not explain their different behavior towards hydrolysis.

\subsection{Acidolysis reactions of grapeseed oil}

\subsubsection{Incorporation degree of capric and caprylic acids}

Six reactions of acidolysis of the grapeseed oil were conducted at 45 ${ }^{\circ} \mathrm{C}$ for $24 \mathrm{~h}$, as previously described (c.f. 2.2.3.). The molar ratio fatty acid/oil of 2:1 corresponds to the stoichiometric value for acidolysis of TAGs when $s n-1,3$ regioselective lipases are used. Table 3 shows the fatty acid profiles of the TAGs obtained by acidolysis of the grapeseed oil with the caprylic or capric acids. Comparing the fatty acid composition of the crude oil (Table 1) with that of the modified TAGs (Table 3), a decrease in the percentage of palmitic (C16:0), stearic (C18:0), oleic (C18:1) and linoleic (C18:2) acids is observed. This decrease is related to the incorporation of the medium chain acids (C8:0 or C10:0), which did not exist in the raw material. Concerning the other fatty acids, little or no variation was observed.

From the composition in fatty acids, the incorporation degree (ID) of capric and caprylic acids in grapeseed oil was calculated. The results are shown in Fig. 1.

ID values for grapeseed oil ranged from $23.62 \pm 1.34$ to $34.53 \pm 0.05 \mathrm{~mol} \%$. For all the biocatalysts tested, higher incorporations were observed for capric acid. The best results were obtained using C10:0 as medium chain fatty acid and Lipozyme RM IM ${ }^{\circledR}$ lipase as catalyst. Nunes et al. (2011), using Lipozyme RM IM ${ }^{\circledR}$ as a catalyst for the acidolysis of olive oil and capric acid, also in solvent-free medium, obtained an incorporation degree of $27.1 \mathrm{~mol} \%$. With Lipozyme TL IM ${ }^{\circ}$ and Novozym $435^{\circ}$, the results of the present study are close to those described by Nunes et al. (2011): $28.8 \mathrm{~mol} \%$ and $30.4 \mathrm{~mol} \%$ of 
Table 3

Fatty acid composition (\%) of modified grapeseed TAGs with caprylic (C8:0) or capric acid (C10:0), by acidolysis catalyzed by Novozym $435^{\circ}$, Lipozyme RM IM ${ }^{\circ}$ or Lipozyme TL IM".

\begin{tabular}{|c|c|c|c|c|c|c|}
\hline \multicolumn{7}{|l|}{ Samples } \\
\hline \multirow{2}{*}{$\begin{array}{l}\text { Fatty acid } \\
(\%)\end{array}$} & \multicolumn{3}{|l|}{ C8:0 } & \multicolumn{3}{|l|}{$\mathrm{C} 10: 0$} \\
\hline & Novozym 435 & Lipozyme TL IM & Lipozyme RM IM & Lipozyme TL IM & Lipozyme RM IM & Novozym 435 \\
\hline C6:0 & $0.22 \pm 0.00$ & - & - & - & - & - \\
\hline C8:0 & $15.55 \pm 0.34$ & $13.90 \pm 0.74$ & $20.05 \pm 1.07$ & $0.08 \pm 0.00$ & $0.01 \pm 0.00$ & - \\
\hline C10:0 & $0.05 \pm 0.00$ & $0.03 \pm 0.00$ & - & $18.49 \pm 0.80$ & $24.61 \pm 0.03$ & $16.65 \pm 0.90$ \\
\hline C14:0 & - & - & $0.02 \pm 0.01$ & $0.03 \pm 0.00$ & $0.02 \pm 0.00$ & - \\
\hline $\mathrm{C} 16: 0$ & $8.80 \pm 0.15$ & $8.97 \pm 0.06$ & $5.93 \pm 0.04$ & $8.03 \pm 0.00$ & $5.55 \pm 0.14$ & $7.97 \pm 0.13$ \\
\hline C16:1 & $0.05 \pm 0.00$ & $0.02 \pm 0.00$ & $0.05 \pm 0.00$ & $0.04 \pm 0.00$ & $0.05 \pm 0.00$ & $0.05 \pm 0.00$ \\
\hline $\mathrm{C} 17: 0$ & - & - & $0.04 \pm 0.00$ & $0.04 \pm 0.00$ & $0.03 \pm 0.00$ & $0.04 \pm 0.00$ \\
\hline $\mathrm{C} 17: 1$ & $0.04 \pm 0.00$ & - & $0.02 \pm 0.00$ & $0.02 \pm 0.00$ & $0.02 \pm 0.00$ & - \\
\hline C18:0 & $3.16 \pm 0.05$ & $3.19 \pm 0.06$ & $2.10 \pm 0.30$ & $2.90 \pm 0.08$ & $1.95 \pm 0.00$ & $2.84 \pm 0.05$ \\
\hline C18:1 & $21.27 \pm 0.37$ & $20.97 \pm 0.98$ & $19.30 \pm 0.18$ & $19.70 \pm 0.25$ & $18.42 \pm 0.01$ & $19.79 \pm 1.01$ \\
\hline C18:2 & $48.37 \pm 0.81$ & $47.30 \pm 1.35$ & $46.90 \pm 0.92$ & $44.45 \pm 0.49$ & $44.16 \pm 0.44$ & $46.17 \pm 0.89$ \\
\hline $\mathrm{C} 18: 3$ & $5.14 \pm 0.09$ & $5.17 \pm 0.06$ & $4.73 \pm 0.05$ & $4.83 \pm 0.18$ & $4.44 \pm 0.00$ & $4.98 \pm 0.25$ \\
\hline C18:3n6 & $0.22 \pm 0.00$ & $0.021 \pm 0.00$ & $0.20 \pm 0.00$ & $0.20 \pm 0.00$ & $0.19 \pm 0.01$ & $0.21 \pm 0.00$ \\
\hline C20:0 & $0.23 \pm 0.00$ & $0.25 \pm 0.01$ & $0.19 \pm 0.00$ & $0.23 \pm 0.02$ & $0.18 \pm 0.00$ & $0.23 \pm 0.01$ \\
\hline C20:1 & $0.27 \pm 0.00$ & $0.31 \pm 0.03$ & $0.31 \pm 0.01$ & $0.30 \pm 0.04$ & $0.29 \pm 0.013$ & $0.33 \pm 0.02$ \\
\hline C21:0 & - & - & $0.01 \pm 0.00$ & $0.02 \pm 0.00$ & $0.01 \pm 0.00$ & - \\
\hline C20:2 & - & $0.02 \pm 0.00$ & - & - & - & - \\
\hline C20:4 & $0.13 \pm 0.02$ & - & - & - & - & - \\
\hline C22:0 & $0.27 \pm 0.02$ & $0.32 \pm 0.02$ & $0.25 \pm 0.00$ & $0.29 \pm 0.03$ & $0.23 \pm 0.01$ & $0.29 \pm 0.01$ \\
\hline $\mathrm{C} 24: 0$ & $0.13 \pm 0.02$ & - & - & - & - & - \\
\hline
\end{tabular}

${ }^{*}$ Mean \pm standard deviation.

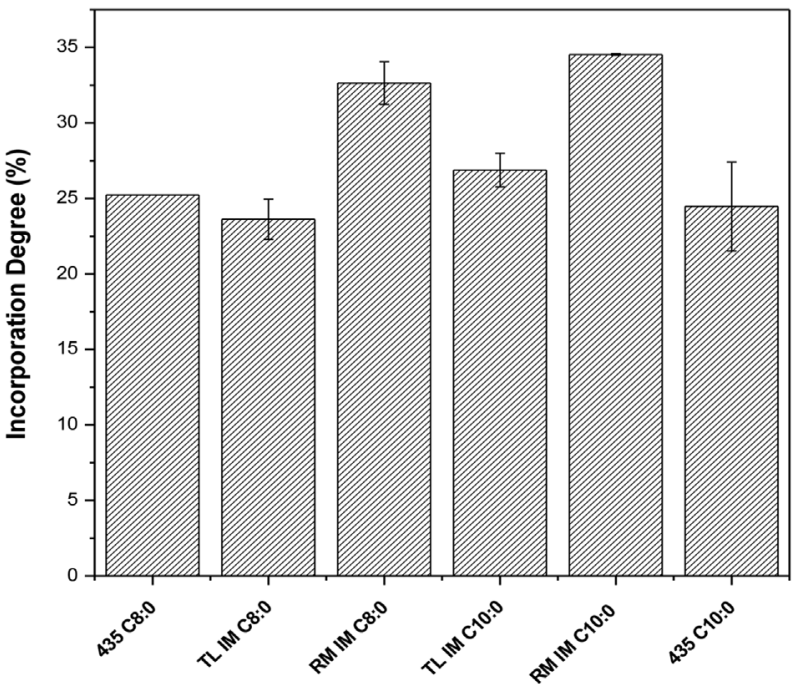

Fig. 1. Incorporation Degree of capric and caprylic acids in grape seed oil.

incorporation, respectively. The acidolysis of grapeseed oil with caprylic or capric acid was also performed in solvent-free media, using non-commercial $s n-1,3$ regioselective lipases namely: the recombinant lipase from Rhizopus oryzae immobilized in Amberlite ${ }^{\mathrm{TM}}$ IRA 96 and Carica papaya lipase self-immobilized in papaya latex (Costa et al., 2018). Yields of new TAGs were between 38 and $69 \mathrm{~mol} \%$, after $48 \mathrm{~h}$ acidolysis at $40{ }^{\circ} \mathrm{C}$, molar ratio fatty acid:oil of $2: 1$.

In our work, Lipozyme RM IM ${ }^{\oplus}$ provided a better incorporation degree $(34.53 \pm 0.05 \mathrm{~mol} \%)$ because, conversely to the other biocatalysts tested (Novozym $435^{\circ}$ and Lipozyme RM IM ${ }^{\circ}$ ), it has both high hydrolytic and esterification activities (Table 2). In fact, acidolysis reaction has been considered as a two-step mechanism reversible reaction of hydrolysis-esterification (Xu, 2003). However, for some lipases, no relationship has been observed between the acidolysis and the hydrolytic activities. This was observed for the recombinant lipase of Rhizopus oryzae immobilized in Eupergit $\mathrm{C}$ and for Carica papaya lipase self-immobilized in the latex, when used as catalysts for the acidolysis reaction aimed at the production of low calorie TAGs or Human Milk Fat Substitutes, respectively (Nunes, Pires-Cabral, Guillén, Valero, \& Ferreira-Dias, 2012; Tecelão, Rivera, Sandoval, \& Ferreira-Dias, 2012).

Moreover, the higher incorporation of capric acid (C10:0) with Lipozyme RM IM ${ }^{\circledR}$ may be due to the longer carbon chain of this acid when compared to caprylic acid (C8:0). The specificity of this lipase is directly related to the size of the fatty acid chain, and the enzyme has a preference for fatty acids with longer carbon chain (Rodrigues \& Fernandez-Lafuente, 2010a; Rodrigues \& Fernandez-Lafuente, 2010b; Caballero et al., 2014). On the contrary, the recombinant lipase of Rhizopus oryzae immobilized in Amberlite IRA 96 showed a preference towards caprylic acid while Carica papaya lipase self-immobilized in papaya latex showed no preference towards caprylic or capric acid (Costa et al., 2018).

\subsubsection{Fatty acid at the sn-2 position in triacylglycerols}

Considering the best result of incorporation degree in the acidolysis reaction of grapeseed oil with C10:0, catalyzed by Lipozyme RM IM", the modified product obtained was evaluated for the fatty acids present at the $s n-2$ position of triacylglycerol.

Table 4

Fatty acid profile at the $s n-2$ position of the grapeseed oil and the modified oil, after 24-h acidolysis catalyzed by Lipozyme RM IM ${ }^{\circ}$.

\begin{tabular}{lll}
\hline Fatty acid & Grapeseed oil & Modified grapeseed oil \\
\cline { 2 - 3 } & $\%$ & $\%$ \\
\hline C10:0 & 0 & 3.36 \\
C16:0 & 2.89 & 2.19 \\
C18:0 & 0.90 & 0.89 \\
C18:1 & 28.15 & 24.33 \\
C18:2 & 63.23 & 63.56 \\
C18:3 & 4.83 & 5.03 \\
$\mathrm{U}^{\mathrm{a}}$ & - & 0.64 \\
Total & 100.00 & 100.00 \\
\hline
\end{tabular}

${ }^{\text {a }} \mathrm{U}=$ unidentified. 
Table 5

Central Composite Rotatable Design (CCRD) used in modeling acidolysis and optimization of reaction conditions as a function of Temperature (T) and acid/ oil molar ratio (MR) (coded and decoded factors).

\begin{tabular}{|c|c|c|c|c|c|c|}
\hline \multirow[t]{2}{*}{ Experiment } & \multirow[t]{2}{*}{ Stage } & \multicolumn{2}{|c|}{ Coded factors } & \multicolumn{2}{|c|}{ Decoded factors } & \multirow{2}{*}{$\begin{array}{l}\text { Response } \\
\text { ID (mol-\%) }\end{array}$} \\
\hline & & $\mathrm{T}$ & $\mathrm{RM}$ & $\begin{array}{l}\mathrm{T} \\
\left({ }^{\circ} \mathrm{C}\right)\end{array}$ & MR (acid:oil) & \\
\hline 1 & Factorial & -1 & -1 & 45 & $2: 1$ & 35.91 \\
\hline 2 & points & -1 & +1 & 45 & $6: 1$ & 42.79 \\
\hline 3 & & +1 & -1 & 65 & $2: 1$ & 34.40 \\
\hline 4 & & +1 & +1 & 65 & $6: 1$ & 54.36 \\
\hline 5 & Axial points & -1.41 & 0 & 41 & $4: 1$ & 41.70 \\
\hline 6 & & +1.41 & 0 & 69 & $4: 1$ & 47.26 \\
\hline 7 & & 0 & -1.41 & 55 & $1: 1$ & 21.29 \\
\hline 8 & & 0 & +1.41 & 55 & $7: 1$ & 48.45 \\
\hline 9 & Central point & 0 & 0 & 55 & $4: 1$ & 38.60 \\
\hline 10 & & 0 & 0 & 55 & $4: 1$ & 43.72 \\
\hline 11 & & 0 & 0 & 55 & $4: 1$ & 41.09 \\
\hline
\end{tabular}

Table 4 shows the results obtained for the determination of fatty acid composition at the $s n-2$ position of the original grapeseed oil and the oil after modification by acidolysis with capric acid, catalyzed by Lipozyme RM IM'.

It can be seen (Table 4) that the $s n-2$ position of triacylglycerols in grapeseed oil mainly contains long chain unsaturated fatty acids (96.2\%). After acidolysis, the compositional fatty acid profile at this position was practically unchanged. In fact, only $3.36 \%$ of capric acid was found at position $s n-2$, which may be related to the acyl migration of this fatty acid. This result was expected, since a regioselective $s n-1,3$ lipase was employed. The obtained results show that Lipozyme RM IM is an adequate biocatalyst for the synthesis of TAGs with the MLM configuration by acidolysis of grapeseed oil.

\subsubsection{Modeling acidolysis reaction}

From the CCRD, it was possible to obtain the incorporation degree for 5 different levels for temperature and molar ratio (MR). Table 5 presents the experimental matrix and the incorporation degree of capric acid in grapeseed oil achieved in each experiment after 24-h acidolysis in solvent-free media, catalyzed by Lipozyme RM IM ${ }^{\oplus}$.

From the obtained results, the main effects and the interaction of the variables temperature and molar ratio on the incorporation degree of capric acid in grapeseed oil were calculated (Table 6).

As can be observed, the molar ratio showed a highly significant positive linear effect $(\mathrm{p}<0.001)$ on the ID of capric acid into grapeseed oil. The positive linear effect of temperature, the positive quadratic effects of both $\mathrm{T}$ an MR and the linear interaction effect showed pvalues higher than 0.05 . However, these effects are important enough to be considered. According to Haaland (1989), it is better to accept factors with effects with $\mathrm{p}$ values higher than 0.05 , rather than to ignore an important factor. This could lead to a considerable decrease in both $\mathrm{R}^{2}$ and $\mathrm{R}_{\text {adj }}^{2}$ of the model fitted to the experimental points.

\section{Table 6}

Linear (L) and quadratic (Q) main effects of temperature (T) and molar ratio (MR), and the interaction T $x$ MR that influenced the incorporation degree of capric acid in grape seed oil, obtained by acidolysis catalyzed by Lipozyme RM IM ${ }^{\infty}$.

\begin{tabular}{lll}
\hline Factor & Effect & $\mathrm{p}$ \\
\hline T (L) & 4.48 & 0.0752 \\
T (Q) & 4.44 & 0.1214 \\
MR (L) & 16.31 & 0.0005 \\
MR (Q) & -5.17 & 0.08201 \\
T X MR & 6.54 & 0.0688 \\
\hline
\end{tabular}

The positive linear effects of temperature and MR on ID indicate that the incorporation degree increases with the values of these factors. The quadratic effect is positive for $\mathrm{T}$ and negative for MR. This indicates that the ID is described by a convex surface, as a function of temperature, and by a concave surface, as a function of the molar ratio. The interaction $\mathrm{T} x \mathrm{MR}$ is positive indicating that an increase in both factors promotes an increase in ID. Fig. 2 shows the three-dimensional response surface, and respective projection, as a function of $\mathrm{T}$ and MR, fitted to the experimental results. As can be seen, it is a saddle-like surface that does not present any optimum points in the experimental region. However, it can be observed that the higher ID of capric acid are observed for temperatures higher than $60-65^{\circ} \mathrm{C}$ and higher molar ratios (higher than 4:1).

Also, in acidolysis optimization, maximum incorporation levels of caprylic acid in sesame oil and in corn oil were expected with a large excess of FFA (MR of 8:1 and 3.9:1 respectively) when Lipozyme RM IM and Lipozyme TL IM were used as catalysts (Kim \& Akoh, 2005; Öztürk, Ustun, \& Aksoy, 2010). In addition, the temperature and reaction time positively affected caprylic acid incorporation (Kim \& Akoh, 2005). Moreover, best results for the incorporation of capric acid in tripalmitin, catalyzed by Lipozyme TL IM were predicted for a molar ratio of 6.5:1 (Bektas, Sevil, Ustun, \& Aksoy, 2008).

In fact, higher molar ratios FFA:oil promote acidolysis by shifting the chemical equilibrium towards product formation. However, for some biocatalysts, an inactivation effect due to the presence of large amounts of free fatty acids may also occur (Nunes, Pires-Cabral, Guillén, Valero \& Ferreira-Dias, 2012; Tecelão et al., 2012).

At industrial-scale, it is important to notice that the use of large amounts of free fatty acids as substrate will increase operation costs in purification process. The unreacted medium-chain fatty acids, which are mixed with the final product, will be recovered by distillation. Increasing the MR also implies an increase in the cost of substrates.

The response surface for the ID (\%) of capric acid in grape seed oil after $24 \mathrm{~h}$ acidolysis, catalyzed by Lipozyme RM IM ${ }^{\circledR}$, can be described by the following second-order polynomial model (equation (2)):

$\mathrm{ID}=105.26-2.87 \mathrm{~T}+0.022 \mathrm{~T}^{2}+0.257 \mathrm{RM}-0.646 \mathrm{RM}^{2}+0.164(\mathrm{~T} x \mathrm{RM})$

The values of the coefficient of determination $\left(\mathrm{R}^{2}\right)$ and the adjusted coefficient of determination ( $R^{2}$ adj) are 0.946 and 0.893 , respectively. This shows a high fit of the models to the experimental results.

After obtaining the statistical model, an experimental test was performed using the reaction conditions that maximized the ID $\left(\mathrm{RM}=7: 1, \mathrm{~T}=69^{\circ} \mathrm{C}\right)$. The ID predicted by the model (equation (2)) was $61.70 \mathrm{~mol} \%$, and the experimentally obtained was $61.50 \mathrm{~mol} \%$. Therefore, this result proves that the model obtained in the present work adequately describes the effect of temperature and molar ratio on the incorporation degree of capric acid in grapeseed oil by acidolysis reaction catalyzed by Lipozyme RM IM ${ }^{\oplus}$.

\section{Conclusions}

From the results obtained, it is possible to conclude that grapeseed oil is an adequate raw material to be used in acidolysis reactions with medium chain acids (C8:0 or C10:0) aimed at producing TAGs of the MLM type rich in linoleic acid, using different commercial biocatalysts (Lipozyme RM IM ${ }^{\circ}$, Lipozyme TL IM ${ }^{\circ}$ and Novozym $435^{\circ}$ ). The best incorporation degree of the medium chain fatty acids was achieved using Lipozyme RM IM ${ }^{\oplus}$ and C10:0 (34.53 \pm 0.05 mol\%) after $24 \mathrm{~h}$ reaction. In addition, with the use of response surface methodology, it was possible to evaluate the influence of molar ratio and temperature on the incorporation degree. The experimental results could be well fitted to a saddle-like surface, where the ID increased with MR and temperature. 

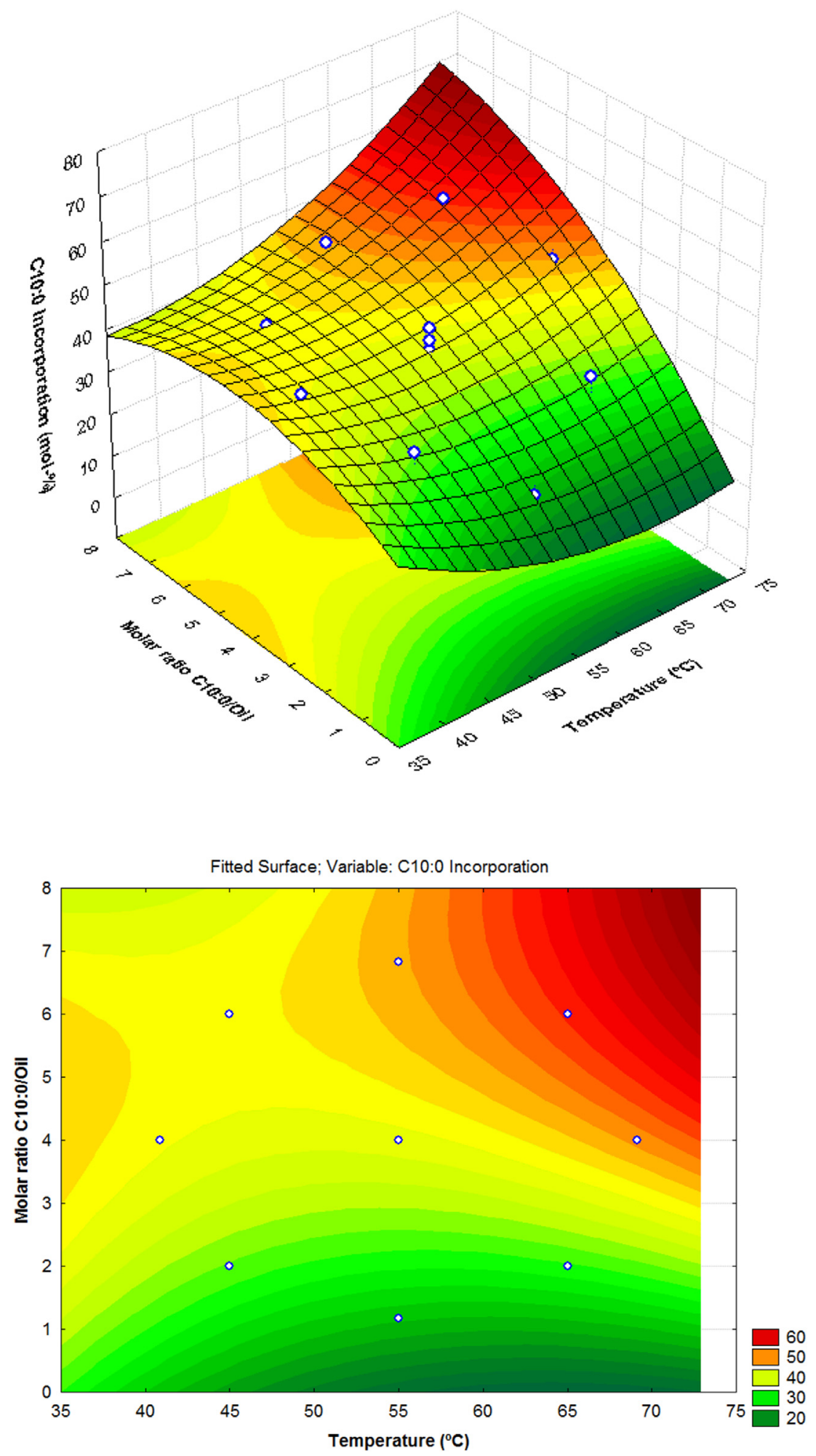

Fig. 2. Response surface (A) and respective projection (B) adjusted for degree of incorporation of capric acid in grapeseed oil, obtained by acidolysis catalyzed by Lipozyme RM IM.

\section{Acknowledgements}

The authors gratefully acknowledge the financial support of (i) Fundação de Amparo à Pesquisa do Estado de São Paulo FAPESP (Process 2017/11482-7), CNPq (Process 446371/2014-9), State University (Unesp, PADC 2014/14-I), Capes, Brazil, (ii) of FCT -
Fundação para a Ciência e a Tecnologia, Portugal, for the funding to the research units of LEAF - Linking Landscape, Environment, Agriculture and Food, (UID/AGR/04129/2013) and MARE -- Marine and Environmental Sciences Centre (UID/MAR/04292/2013) and to (iii) Novozymes A/S (Bagsvaerd, Denmark) for the gift of lipases. 


\section{References}

AOCS (2011). Official methods and recommended practices (6th ed.). American Oil Chemists' Society: AOCS Press.

Bektas, I., Sevil, Y., Ustun, G., \& Aksoy, H. A. (2008). Production of reduced calorie structured lipid by acidolysis of tripalmitin with capric acid: Optimisation by response surface methodology. Journal of the Science of Food and Agriculture, 88, 1927-1931.

Bowen, J. K., Kris-Etherton, M. P., Shearer, C. G., West, G. S., Reddivari, L., \& Jones, H. J. P. (2017). Oleic acid-derived oleoylethanolamide: A nutritional science perspective. Progress in Lipid Research, 67, 1-15.

Caballero, E., Soto, C., Olivares, A., \& Altamirano (2014). Potential use of avocado oil on Structured lipids MLM type production catalysed by commercial immobilised lípases. PLoS One, 9, 1-7.

Cao, Y., Qi, S., Zhang, Y., Wang, X., Yang, B., \& Wang, Y. (2013). Synthesis of Structured lipids by Lipase-Catalyzed interesterification of triacetin with camellia oil methyl esters and preliminary evaluation of their plasma lipid-lowering effect in mice. Molecules, 18, 3733-3744.

Casas - Godoy, L., Marty, A., Sandoval, G., \& Ferreira - Dias, S. (2013). Optimization of medium chain length fatty acid incorporation into olive oil catalyzed by immobilized Lip2 from Yarrowia lipolytica. Biochemical Engineering Journal, 77, 20-27.

Codex (1999). Codex standard for named vegetable oils, CX-STAN 210.

Costa, C. M., Canet, A., Rivera, I., Osório, N. M., Sandoval, G., Valero, F., et al. (2018) Production of MLM type structured lipids from grape seed oil catalyzed by noncommercial lipases. European Journal of Lipid Science and Technology. https://doi.org/ 10.1002/ejlt.201700320 in press.

Da Porto, C., \& Natolino, A. (2017). Supercritical fluid extraction of polyphenols from grape seed (Vitis vinifera): Study on process variables and kinetics. The Journal of Supercritical Fluids, 130, 239-245.

Dhakal, H. K., Jung, H. K., Chae, H. L., Shannon, G. J., \& Lee, D. J. (2014). Variation of unsaturated fatty acids in soybean sprout of high oleic acid accessions. Food Chemistry, 164, 70-73.

Duba, S. K., \& Fiori, L. (2015). Supercritical $\mathrm{CO}_{2}$ extraction of grape seed oil: Effect of process, parameters on the extraction kinetics. The Journal of Supercritical Fluids, 98, 33-43.

Durante, M., Motefusco, A., Marrese, P. P., Soccio, M., Pastore, D., Piro, G., et al. (2017). Seeds of pomegranate, tomato and grapes: An underestimated source of natural bioactive molecules and antioxidants from agri-food by-products. Journal of Food Composition and Analysis, 63, 65-72.

FAO (1999). Codex standards for fats and oils from vegetable sources.

Ferreira-Dias, S., Sandoval, G., Plou, F., \& Valero, F. (2013). The potential use of lipases in the production of fatty acid derivatives for the food and nutraceutical industries. Electronic Journal of Biotechnology, 16, 1-38.

Fiori, L., Lavelli, V., Duba, S. K., Harsha, S. C. S. P., Mohamed, B. H., \& Guella, G. (2014). Supercritical CO 2 extraction of oil from seeds of six grape cultivars: Modeling of mass transfer kinetics and evaluation of lipid profiles and tocol contents. The Journal of Supercritical Fluids, 94, 71-80.

Glampedaki, P., \& Dutschk, V. (2014). Stability studies of cosmetic emulsions prepared from natural products such as wine, grape seed oil and mastic resin. Colloids and Surfaces A: Physicochemical and Engineering Aspects, 460, 306-311.

Haaland, P. D. (1989). In D. B. Owen (Ed.). Experimental design in biotechnology, statistics: Textbooks and monographs (pp. 258). New York (United States of America): Marcel Dekker Inc.

Irandoost, P., Ebrahimi-Mameghani, M., \& Pirouzpanah, S. (2013). Does grape seed oil improve inflammation and insulin resistance in overweight or obese women? International Journal of Food Sciences and Nutrition, 64, 706-710.

ISO 12966-2 (2011). Animal and vegetable fats and oils - gas chromatography of fatty acid methyl esters - Part 2: Preparation of methyl esters of fatty acids. International organization for standardization.

Kim, B. H., \& Akoh, C. C. (2005). Modelling of lipase-catalyzed acidolysis of sesame oil and caprylic acid by response surface methodology: Optimization of reaction conditions by considering both acyl incorporation and migration. Journal of Agricultural and Food Chemistry, 53, 8033-8037.

Li, X., Peng, X., Wang, Q., Zuo, H., Meng, X., \& Liu, B. (2017). Effective detoxification of patulin from aqueous solutions by immobilized porcine pancreatic lipase. Food Chemistry, 78, 48-56.

Liu, S., Dong, X., Wei, F., Wang, X., Lv, X., Zhong, J., et al. (2015). Ultrasonic pretreatment in lipase-catalyzed synthesis of Structured lipids with high 1,3-dioleoyl-2-palmitoylglycerol content. Ultrasonics Sonochemistry, 23, 100-108.

Mironeasa, S., Leahua, A., Codinăa, G. G., Stroea, G. S., \& Mironeasa, C. (2010). Grapeseed: Physicochemical, structural characteristic and oil content. Journal of Agroalimentary Process and Technologies, 16, 1-6.

Morales-Medina, R., Munio, M., Guadix, A., \& Guadix, M. E. (2017). Development of an up-grading process to produce MLM Structured lipids from sardine discards. Food Chemistry, 228, 634-642.

Nunes, P. A., Pires-Cabral, P., \& Ferreira-Dias, S. (2011). Production of olive oil enriched with medium chain fatty acids catalysed by commercial immobilised lipases. Food Chemistry, 127, 993-998.

Nunes, P. A., Pires-Cabral, P., Guillén, M., Valero, F., \& Ferreira-Dias, S. (2012). Optimized production of MLM triacylglycerols catalyzed by immobilized heterologous Rhizopus oryzae lipase. Journal of the American Oil Chemists's Society, 89(7), 1287-1295.

Öztürk, T., Ustun, G., \& Aksoy, H. A. (2010). Production of medium-chain triacylglycerols from corn oil: Optimization by response surface methodology. Bioresource Technology, 101, 7456-7461.

Paula, A. V. (2012). Reestruturação da gordura de leite por interesterificação enzimática empregando lipase imobilizada: otimização das condições reacionais e operacionais. Lorena: Escola de Engenharia de Lorena, Universidade de São Paulo212.

Pinto, M. C. C., Freire, D. M. G., \& Pinto, J. C. (2014). Influence of the morphology of core-shell supports on the immobilization of Lipase B from Candida antarctica. Molecules, 19, 12509-12530.

Raeisi, M., Tajik, H., Aliakbarlu, J., Mirhosseini, H. S., \& Hosseini, H. M. S. (2015). Effect of carboxymethyl cellulose-based coatings incorporated with Zataria multiflora Boiss. essential oil and grapeseed extract on the shelf life of rainbow trout fillets. LWT - Food Science and Technology, 64, 898-904.

Rehman, S., Wang, P., Bhatti, N. H., Bilas, M., \& Asgher, M. (2017). Improved catalytic properties of Penicillium notatum lipase immobilized in nanoscale silicone polymeric films. International Journal of Biological Macromolecules, 97, 279-286.

Rodrigues, R. C., \& Fernandez-Lafuente, R. (2010a). Lipase from Rhizomucor miehei as an industrial biocatalyst in chemical process. Journal of Molecular Catalysis B: Enzymatic, $64,1-22$.

Rodrigues, R. C., \& Fernandez-Lafuente, R. (2010b). Lipase from Rhizomucor miehei as a biocatalyst in fats and oils modification. Journal of Molecular Catalysis B: Enzymatic, $66,15-32$.

Rombaut, N., Savoire, R., Thomasset, B., Bélliard, T., Castelli, J., Hecke, V. E., et al. (2014). Grape seed oil extraction: Interest of supercritical fluid extraction and gasassisted mechanical extraction for enhancing polyphenol co-extraction in oil. Comptes Rendus Chimie, 17, 284-292.

Tecelão, C., Rivera, I., Sandoval, G., \& Ferreira-Dias, S. (2012). Carica papaya latex: A lowcost biocatalyst for human milk fat substitutes production. European Journal of Lipid Science and Technology, 114(3), 266-276.

Viladomiu, M., Hontecillas, R., \& Bassaganya-Riera, J. (2016). Modulation of inflammation and immunity by dietary conjugated linoleic acid. European Journal of Pharmacology, 785, 87-95.

Wang, Y., Xia, L., Xu, X., Xie, L., \& Duan, Z. (2012). Lipase-catalyzed acidolysis of canola oil with caprylic acid to produce medium-, long- and medium-chain-type Structured lipids. Food and Bioproducts Processing, 90, 707-712.

$\mathrm{Xu}, \mathrm{X}$. (2003). Engineering of enzymatic reactions and reactors for lipid modification and synthesis. European Journal of Lipid Science and Technology, 105, 289-304.

Yang, B., Chen, H., Stanton, C., Ross, P. R., Zhang, H., Chen, Q. Y., et al. (2015). Review of the roles of conjugated linoleic acid in health and disease. Journal of Functional Foods, 15, 314-325. 